\section{Color-selective adaptation in contrast thresholds for detecting the form but not the motion in moving gratings}

\author{
WILLIAM LOVEGROVE and PAUL EVANS \\ University of Tasmania \\ Hobart, Australia 7001
}

It has been demonstrated that there are two phases differing in spatial and temporal properties in the classical motion aftereffect (Bonnet, Bouvier, \& Petiteau, 1976; Bonnet \& Pouthas, 1972; Favreau, 1976; Taylor, 1963). Lovegrove, Mapperson, and Bowling (1980) investigated these two phases by varying the interstimulus interval between inspection and test stimuli. They demonstrated that while the magnitude of aftereffect in Phase 1 was uninfluenced by the relative color of inspection and test stimuli, the magnitude of Phase 2 was significantly greater when inspection and test stimuli were viewed in the same colored light than when they were viewed in different colored light under monoptic viewing conditions. It was argued (Lovegrove, Over, \& Broerse, 1979; Lovegrove et al., 1980) that the properties of these two phases of the motion aftereffect correspond with the properties of transient and sustained channels identified by both physiological (Cleland, Dubin, \& Levick, 1971; Enroth-Cugell \& Robson, 1966; Gouras, 1974) and psychological evidence (Kulikowski \& Tolhurst, 1973; King-Smith \& Kulikowski, 1975; Tolhurst, 1977).

There are two distinct contrast thresholds in the perseption of such stimuli as flickering gratings (KingSmith \& Kulikowski, 1975), moving gratings (Hess, Howell, \& Kitchin, 1978), and moving spirals (Richards, 1971). One threshold is for the detection of movement without form, and the other for the detection of form in the moving stimulus. The general argument is that movement thresholds are mediated by transient cells and form thresholds by sustained cells.

Color-motion processing in the human visual system has previously been investigated using luminance threshold measures by Mayhew (1973), who showed color selectivity in direction-specific adaptation. Mayhew (1973) interpreted his data as evidence for the existence of neural processes selective for both color and motion, although he did not consider his results in terms of possible sustained-transient involvement. The use of a threshold measure combined with the data on separate motion and form thresholds for moving gratings provides a further means of testing the hypothesis proposed by Lovegrove et al. (1980). If Phases 1 and 2 of the motion aftereffect do reflect adaptation of the color-insensitive transient and the color-sensitive sustained cells, respectively, it is predicted that following adaptation to a colored moving grating the elevation in the form threshold of a moving test grating would depend on the color relationship between the inspection and test stimuli, whereas elevation of the motion threshold would not. The present experiment investigates this prediction.

\section{METHOD}

\section{Subjects}

Twelve subjects with normal or corrected-to-normal vision participated in this experiment. All subjects were screened for abnormalities in color vision on the Ishihara test for color blindness.

\section{Apparatus}

The square-wave moving gratings used as inspection and test stimuli were produced by attaching black tape to a motor-driven belt. The circular inspection and test stimuli subtended $4 \mathrm{deg} 18 \mathrm{~min}$ and $3 \mathrm{deg} 6 \mathrm{~min}$ in diameter, respectively, and were presented in a Scientific Prototype three-field tachistoscope (Model GB). The inspection grating had a Michelson contrast of .6, a spatial frequency of 1.1 cycles/deg, a velocity of 6 cycles/sec and spaceaverage luminance of $1.1 \mathrm{~cd} / \mathrm{m}^{2}$. The same stimulus at reduced contrast was used as the test stimulus. Both inspection and test stimuli could be displayed in red (Wratten filter 26; dominant wavelength $620.6 \mathrm{~nm}$ in illuminant A) and green (Wratten filter 55 ; dominant wavelength $524.1 \mathrm{~nm}$ in illuminant A) light. The Wratten filters were transillumunated by the tachistoscope light source. The contrast of the test stimuli was controlled by means of rotating polarizing filters of opposite polarity placed in front of the moving grating and uniform fields matched in wavelength and space-average luminance with the grating field. Luminance of the test field was held constant by superimposing red and green light from the blank field and Field 2, respectively, of the tachistoscope onto the grating field. Contrast readings (Richards, 1971) were in terms of degrees (to the nearest half degree).

\section{Procedure}

Data were collected in two experimental sessions. In each session, thresholds for either movement or form in red and green light were determined before and after adaptation to a moving grating. Half the subjects adapted to moving gratings in green light and the other half to red light. Motion thresholds were determined in the first session for half the subjects and form thresholds in the first session for the other half. Motion thresholds were determined by the ascending method of adjustment until motion could just be seen. Form thresholds were determined in the same manner, except that the subject rotated the polarizing filter until the bars of the moving grating were also visible. Trials were always started below threshold to eliminate any but the main adaptation effects. A practice session using achromatic stimuli was given to each subject before the start of the first experimental session. Pretest data show that the experimental conditions used produced separate form and motion thresholds for each subject. Eight pre- and posttest measures (four in both green and red) were obtained within a session. Order of presentation of test stimulus colors was alternated within a subject and counterbalanced across subjects. The first posttest measure was taken after $4 \mathrm{~min}$ adaptation and the other seven each after a 1-min adaptation period. A 5-sec interstimulus interval, during which subjects closed their eyes, was used to allow for changes of the Wratten filters. Testing was carried out in a darkened room, and viewing was monocular. 


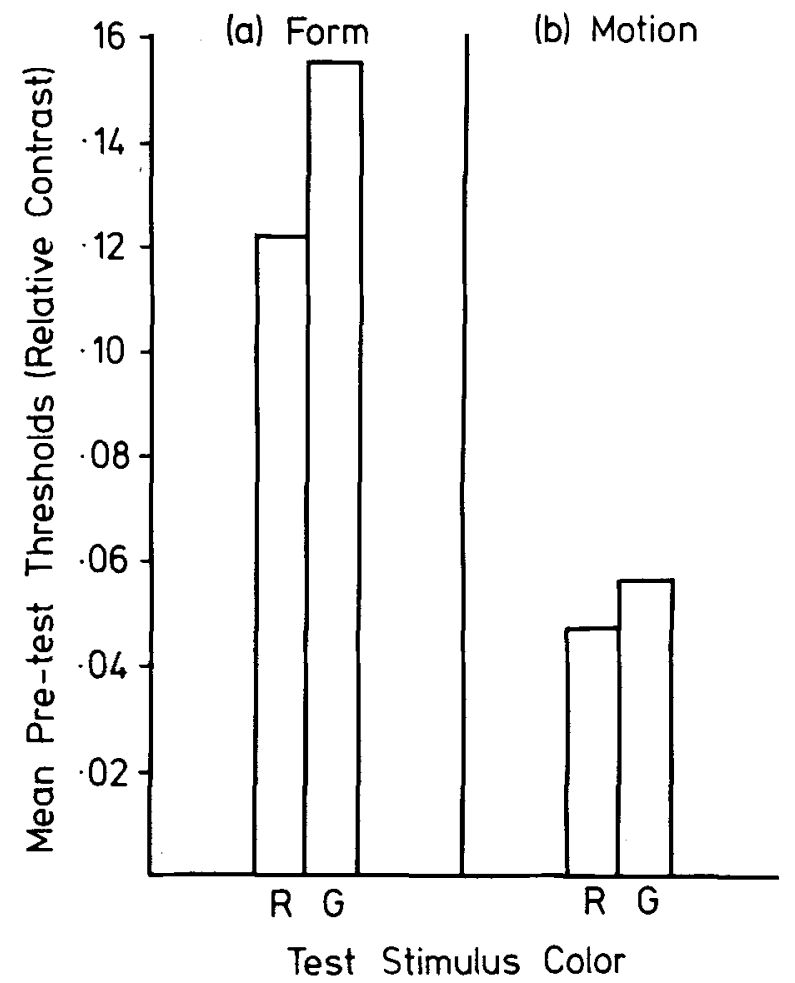

Figure 1. Mean pretest form and motion thresholds (in relative contrasi) of the moving grating in red and green light.

\section{RESULTS}

All scores were converted to relative contrast scores using the procedure outlined by Richards (1971). Figure 1 shows the mean form and motion thresholds in red and green light for the pretest settings. An analysis of variance revealed that target color had a significant effect $[F(1,11)=22.54, p<.01]$ : target thresholds were lower in red than in green light. There was also a significant effect $[F(1,11)=31.16$, $p<.01]$ due to threshold type (form and motion) with motion thresholds being lower than form thresholds. This supports previous results (Hess et al., 1978; King-Smith \& Kulikowski, 1975;) and indicates that the present experimental conditions were suitable for separating thresholds for sustained and transient channels. In addition, there was a significant interaction $[\mathrm{F}(1,11)=13.2, \mathrm{p}<.01]$ between target color (red-green) and threshold type (form-motion). This interaction results from the finding that form thresholds are lower in red than in green light but motion thresholds are the same in both colors.

The mean form and motion threshold elevation effects (the difference between pre- and postsettings) for each color combination are shown in Figure 2. A three-way analysis of variance revealed no significant effect due to inspection stimulus color $[\mathrm{F}(1,5)=.25$, $p>.05]$ but did reveal a significant effect due to test stimulus color $[F(1,5)=5.85, p<.05]$. The latter results from greater threshold change occurring with green test stimuli. There was a significant effect $[F(1,5)=8.11, p<.01]$ of target type (form or motion), indicating that form thresholds were elevated more after adaptation than were motion thresholds. The interaction between inspection and test colors was significant $[F(1,5)=11.14, p<.01]$, indicating that the magnitude of the change in threshold following adaptation depended on the color relationship between inspection and test stimuli. Same color inspection and test stimuli combinations produced greater threshold changes than different color combinations. Neither inspection color $[F(1,5)=.13 . p>.05]$ nor the test color $[F(1,5)=.56, p>.05]$ interacted significantly with threshold type (form or motion). There was, however, a significant three-way interaction between inspection color, test color, and threshold type $[\mathrm{F}(1,5)=6.22, \mathrm{p}<.05]$. Inspection of Figure 2 reveals that this interaction results from the form (Figure $2 \mathrm{a}$ ) but not the motion (Figure $2 \mathrm{~b}$ ) thresholds being dependent upon the color relationship between inspection and test stimuli.

\section{DISCUSSION}

The pretest scores provide further indirect evidence for motion thresholds (under the conditions used in this experiment) being determined by color-insensitive mechanisms. By contrast, the finding that form thresholds are lower in red illumination than in green

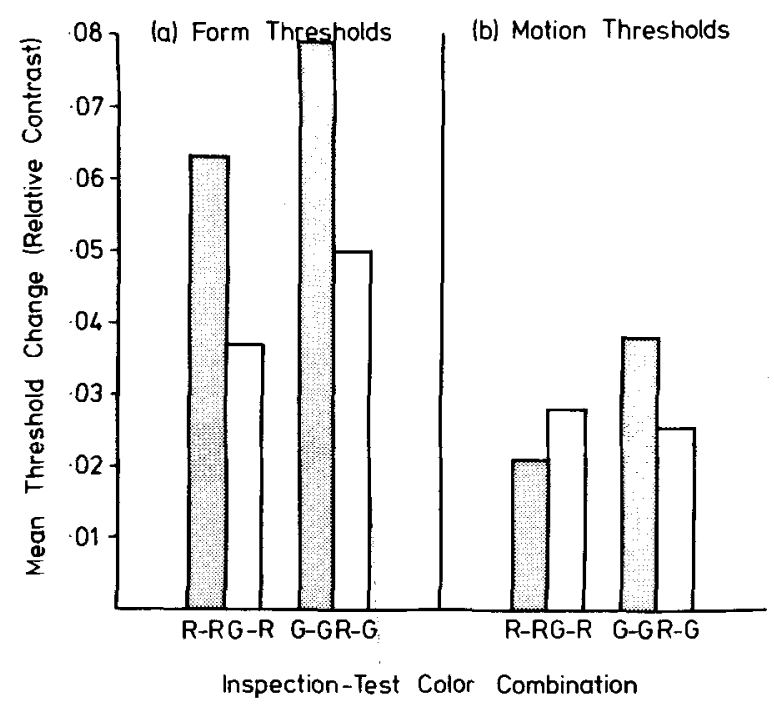

Figure 2. Mean contrast threshold change following adaptation for each combination of inspection and test stimulus color for form (Figure 2a) and motion (Figure 2b) thresholds. $R$ signifies red and $G$, green. In each case, the inspection stimulus color is shown first. 
further suggest a link between form and color processing.

The adaptation results show clearly that the threshold for form is significantly influenced by the color relationship between the inspection and test stimuli, whereas the motion threshold for the same test stimulus is uninfluenced by this relationship. These findings are consistent with an explanation of motion thresholds in terms of color-insensitive transient channels and form thresholds in terms of color-sensitive sustained channels. Both systems are adapted during inspection of moving colored gratings and remain so for some time afterwards. The results corroborate the explanation of two phases of the motion aftereffect in terms of adaptation of both sustained and transient channels. In addition, the present results demonstrating color selectivity in form threshold measures following adaptation to only one direction of motion in one color are consistent with the recent argument (Lovegrove et al., 1979; Lovegrove et al., 1980) that colorcontingent aftereffects can be demonstrated following adaptation to single color-motion combinations as well as following adaptation to double color-motion combinations which have been more widely used (Favreau, Emerson, \& Corballis, 1972; Hepler, 1968; Mayhew \& Anstis, 1972; Stromeyer \& Mansfield, 1970).

There have been explanations of the general class of spatial-chromatic aftereffects in terms of learning theories (e.g., Murch, 1976; Skowbo, Gentry, Timney, \& Morant, 1974). The present evidence combined with that on phases in the motion aftereffect links these effects closely to the properties of sustained and transient channels. To the extent that further research supports this link, general theories of spatialchromatic aftereffects should consider the possible physiological basis of such effects.

\section{REFERENCES}

Bonnet, C., Bouvier, A., \& Petiteau, H. Phases in movement aftereffects and their relationship to the kinetic figural aftereffect. Psychological Research, 1976, 38, 267-282.

Bonnet, C.. \& Pouthas, V. Interactions between spatial and kinetic dimensions in movement aftereffects. Perception \& Psychophysics, 1972, 12, 193-200.
Clenand, B. G., Dubin, M. W., \& Levick, W. R. Sustained and transient neurones in the cat's retina and lateral geniculate nucleus. Journal of Physiology, 1971, 217, 473-496.

Enroth-Cugell, C., \& Robson, J. G. The contrast sensitivity of retinal ganglion cells of the cat. Journal of Physiology, 1966, $187,517-552$.

FAVREAU, $O$. Interference in color-contingent motion aftereffects. Quarterly Journal of Experimental Psychology, 1976, 28, $553-560$.

Favmeav, O., Emerson, V., \& Corballis, M. C. Movement aftereffects contingent on color. Science, 1972, 176, 78-79.

Gouras, P. Opponent color cells in different layers of foveal striate cortex. Journal of Physiology, 1974, 238, 583-602.

HEPLER, N. Color: A motion-contingent aftereffect. Science, 1968, 162, 376-377.

Hess, R. F., Howell, E. R., \& KrTchin, J. E. On the relationship between pattern and movernent perception in strabismic amblyopia. Vision Research, 1978, 18, 375-377.

King-Smith, P. E., \& Kulikowskt, J. J. Pattern and flicker detection analysed by subthrestiold summation. Journal of Physiology, 1975, 249, 519-548.

Kulıkowski, J. J., \& Tolhurst, D. J. Psychophysical evidence for sustained and transient detectors in human vision. Journol of Physiology, 1973, 232, 149-162.

Lovegrove, W. J., Over, R., \& Broense, J. Color selectivity in motion aftereffect. Perception \& Psychophysics, 1979, 25, 157-159.

Lovegrove, W. J., Mapperson, B., \& Bowling, A. Presence and absence of color selectivity in the motion aftereffect. Perception \& Psychophysics, 1980, 27, 33-36.

MAYHEW. J. W. Luminance thresholds for motion contingent on color. Perception, 1973, 2, 4!-5!.

Mayhew, J. E. W., \& Anstis, S. M. Movement aftereffects contingent on color, intensity, and pattern. Perception \& Psychophysics, 1972, 12, 77-85.

Munch, G. M. Classical conditioning of the McCollough effect. Temporal parameters. Vision Research, 1976, 16, 615-619.

RICHARDS, W. Motion detection in man and other animals. Brain, Behaviour and Evolution, 1971, 4, 162-181.

Skowbo, D., Gentry, T. A., Timney, B. N., \& Morant, B. B. The McCollough effect: Influence of several kinds of visual stimulation on decay rate. Perception \& Psychophysics, 1974, $16,47-49$.

Stromeyer, C. F, \& Mansfield, R. Colored aftereffects produced with moving edges. Perception \& Psychophysics, 1970. 7, 108-114.

TAYLOR, M. M. Tracking the decay of the aftereffect of seen rotary movement. Perceptual and motor Skills, 1963, 16, 119-129.

TolmursT, D. J. Color-coding properties of sustained and transient cells in human vision. Nature, 1977, 266, 266-267.

(Received for publication February 29, 1980; acceoted March 10. 1980.) 